\title{
Urban-rural differences in the associated factors of severe under- 5 child undernutrition based on the composite index of severe anthropometric failure (CISAF) in Bangladesh
}

Asibul Islam Anik ${ }^{1,2+}$, Mohammad Rocky Khan Chowdhury ${ }^{3+}$, Hafiz T. A. Khan ${ }^{4}$, Md Nazrul Islam Mondal ${ }^{5}$, Nirmala K. P. Perera ${ }^{6,7}$ and Manzur Kader ${ }^{8^{*}}$

\begin{abstract}
Introduction: Severe undernutrition among under-5 children is usually assessed using single or conventional indicators (i.e., severe stunting, severe wasting, and/or severe underweight). But these conventional indicators partly overlap, thus not providing a comprehensive estimate of the proportion of malnourished children in the population. Incorporating all these conventional nutritional indicators, the Composite Index of Severe Anthropometric Failure (CSIA F) provides six different undernutrition measurements and estimates the overall burden of severe undernutrition with a more comprehensive view. This study applied the CISAF indicators to investigate the prevalence of severe under-5 child undernutrition in Bangladesh and its associated socioeconomic factors in the rural-urban context.

Methods: This study extracted the children dataset from the 2017-18 Bangladesh Demographic Health Survey (BDHS), and the data of 7661 children aged under-5 were used for further analyses. CISAF was used to define severe undernutrition by aggregating conventional nutritional indicators. Bivariate analysis was applied to examine the proportional differences of variables between non-severe undernutrition and severe undernutrition group. The potential associated socioeconomic factors for severe undernutrition were identified using the adjusted model of logistic regression analysis.
\end{abstract}

\footnotetext{
* Correspondence: Manzur.Kader@ki.se

${ }^{\dagger}$ Asibul Islam Anik and Mohammad Rocky Khan Chowdhury are jointly first authors.

${ }^{8}$ Unit of Occupational Medicine, Institute of Environmental Medicine |

Karolinska Institutet, Solnavägen 4, Torsplan floor 10, 11365 Stockholm,

Sweden

Full list of author information is available at the end of the article
}

(c) The Author(s). 2021 Open Access This article is licensed under a Creative Commons Attribution 4.0 International License, which permits use, sharing, adaptation, distribution and reproduction in any medium or format, as long as you give appropriate credit to the original author(s) and the source, provide a link to the Creative Commons licence, and indicate if changes were made. The images or other third party material in this article are included in the article's Creative Commons licence, unless indicated otherwise in a credit line to the material. If material is not included in the article's Creative Commons licence and your intended use is not permitted by statutory regulation or exceeds the permitted use, you will need to obtain permission directly from the copyright holder. To view a copy of this licence, visit http://creativecommons.org/licenses/by/4.0/ The Creative Commons Public Domain Dedication waiver (http://creativecommons.org/publicdomain/zero/1.0/) applies to the data made available in this article, unless otherwise stated in a credit line to the data. 
Results: The overall prevalence of severe undernutrition measured by CISAF among the children under-5 was $11.0 \%$ in Bangladesh (rural $11.5 \%$ vs urban 9.6\%). The significant associated socioeconomic factors of severe undernutrition in rural areas were children born with small birth weight (AOR: 2.84), children from poorest households (AOR: 2.44), and children aged $<36$ months, and children of uneducated mothers (AOR: 2.15). Similarly, in urban areas, factors likechildren with small birth weight (AOR: 3.99), children of uneducated parents (AOR: 2.34), poorest households (APR: 2.40), underweight mothers (AOR: 1.58), mothers without postnatal care (AOR: 2.13), and children's birth order $\geq 4$ (AOR: 1.75), showed positive and significant association with severe under-5 undernutrition.

Conclusion: Severe undernutrition among the under-5 children dominates in Bangladesh, especially in rural areas and the poorest urban families. More research should be conducted using such composite indices (like- CISAF) to depict the comprehensive scenario of severe undernutrition among the under- 5 children and to address multi-sectoral intervening programs for eradicating severe child undernutrition.

Keywords: CIAF, CISAF, Severe child undernutrition, Maternal \& child health, Urban-rural disparity, Bangladesh

\section{Introduction}

Undernutrition is one of the common causes of morbidity and mortality in children. Around 195 million children under-5 suffer from any form of undernutrition across the world $[1,2]$. About half of all fatalities in children under-5 are attributable to undernutrition [3]. Asia is the home to over $70 \%$ of the world's malnourished children, with India, Pakistan, and Bangladesh having the highest prevalence in this region [4]. In 2014, over $40 \%$ of Bangladeshi children under- 5 experience undernutrition, with $18 \%$ experiencing severe undernutrition [5]. Children with severe undernutrition experience short- and long-term adverse physiological outcomes, such as poor physical growth, morbidity, inadequate cognitive development, and physical incapacity [6].

Undernutrition affects children for the rest of their lives, and its consequences are passed down from generation to generation. Since undernutrition is more prevalent among rural female children, these undernourished female children become stunted mothers in future. Then by giving birth to a stunted child, the circle continues to revolve [7]. Among the other South Asian countries, though Bangladesh made a significant achievement in declining the prevalence rate of stunting children from 1997 (60\%) to 2018 (31\%) [8], it is still inadequate to attain the target of ending all forms of undernutrition by 2030 (Sustainable Development Goal- 2, Target 2.2) [9]. There are considerable urban-rural disparities in terms of stunting in Bangladesh, with a higher prevalence among rural (33\%) than urban (25\%) children [8].

Along with the urban-rural disparities, other associated factors for undernutrition are shaped by people's complex interactions with their social, cultural, economic, and environmental contexts. Social determinants of health provide a framework for understanding the inequalities in health risks and outcomes within and between populations [10]. Improper postnatal care (i.e., inadequate infant and young child feeding practices and inadequate access to health care); children's low birth weight, frequent infections; parent's least wealthy socioeconomic status (i.e., lower education level, unemployment, and poor wealth-index); and other household characteristics (i.e., unsafe drinking water, and lack of access to sanitation), etc. were found to be strongly associated with under-5 child undernutrition in different studies [5, 11-13]. However, current evidence on severe under- 5 child undernutrition focused on single or may not provide a convincing (i.e., severe stunting, severe wasting, and/or severe underweight) and economic dimensions at single time points. But these conventional indicators partly overlap, thus not providing a comprehensive estimate of the proportion of malnourished children in the population [14].

By incorporating all these conventional nutritional indicators, the Composite Index of Anthropometric Failure (CIAF) provides six different undernutrition measurements and estimates the overall burden of undernutrition. Moreover, the CIAF accurately depicts a single aggregate proportion of all malnourished children in a population [14]. On the other hand, Vollmer et al. (2017) introduced an updated version of the CIAF, i.e., Composite Index of Severe Anthropometric Failure (CISAF), which provides a more comprehensive view of the extent and pattern of associated factors of severe undernutrition in resource-poor settings [15]. As per the CIAF, undernutrition was more prevalent among rural children (54\%) than urban children (45\%) in Bangladesh. Irrespective of urban-rural differences, sociodemographic characteristics of children, such as older age, mothers' illiteracy, underweight mothers, and poorest wealth-index showed positive association with undernutrition $[16,17]$. But rarely any study has applied the CISAF to explore the prevalence and the intricate interaction of individual, community, public policy, and environmental characteristics associated with severe under-5 child undernutrition in Bangladesh. Addressing this knowledge gap, this study used the CISAF to investigate the 
prevalence and associated socioeconomic factors of severe under-5 child undernutrition in Bangladesh's rural and urban areas.

\section{Methodology}

We analysed the children dataset (BDKR7RDT) from 2017 to 18 of the Bangladesh Demographic Health Survey (BDHS). The average response rate was $99 \%$. The BDHS uses two-stage stratified sampling techniques to select primary sampling units (PSUs) and households. During the first stage, PSUs or enumeration areas (EA) were designed based on the census survey 2011 conducted by the Bangladesh Bureau of Statistics. The probability proportional to the EA size technique was used to select PSUs. During the second stage, an equal probability systematic sampling technique was used to determine households from PSUs. Reproductive-aged women (1549 years) were interviewed from each household. The BDHSs collect data on social and demographic factors, health, and nutritional factors from adults (male and female) residing in non-institutional dwellings. A standard questionnaire was used for data collection. For details of the survey questionnaire, sample design, data collection procedure (see BDHS reports 2017-18) [8]. The BDHS 2017-18 collected anthropometric data from 8759 under- 5 children, and data from 7661 children were analysed (Fig. 1).

\section{Outcome variables}

Severe nutritional indicators for under-5 children were categorised into seven groups: (A) no severe failure; (B) severe wasting only; $(C)$ severe wasting and severe underweight; (D) severe wasting and severe stunting and severe underweight; (E) severe stunting and severe underweight; (F) severe stunting only; and (G) severe underweight only (Table 1). A child is considered having severe undernutrition if she/he has any anthropometric failure from B to G (Fig. 2).

The primary outcome measure was severe under-5 child undernutrition using the CISAF. A child was considered to be severely stunted and severely wasted and severely underweight if the $\mathrm{z}$-scores of length/height-forage, weight-for-height/length, and weight-for-age were below minus three (-3.0) Standard Deviations (SD) (i.e.,

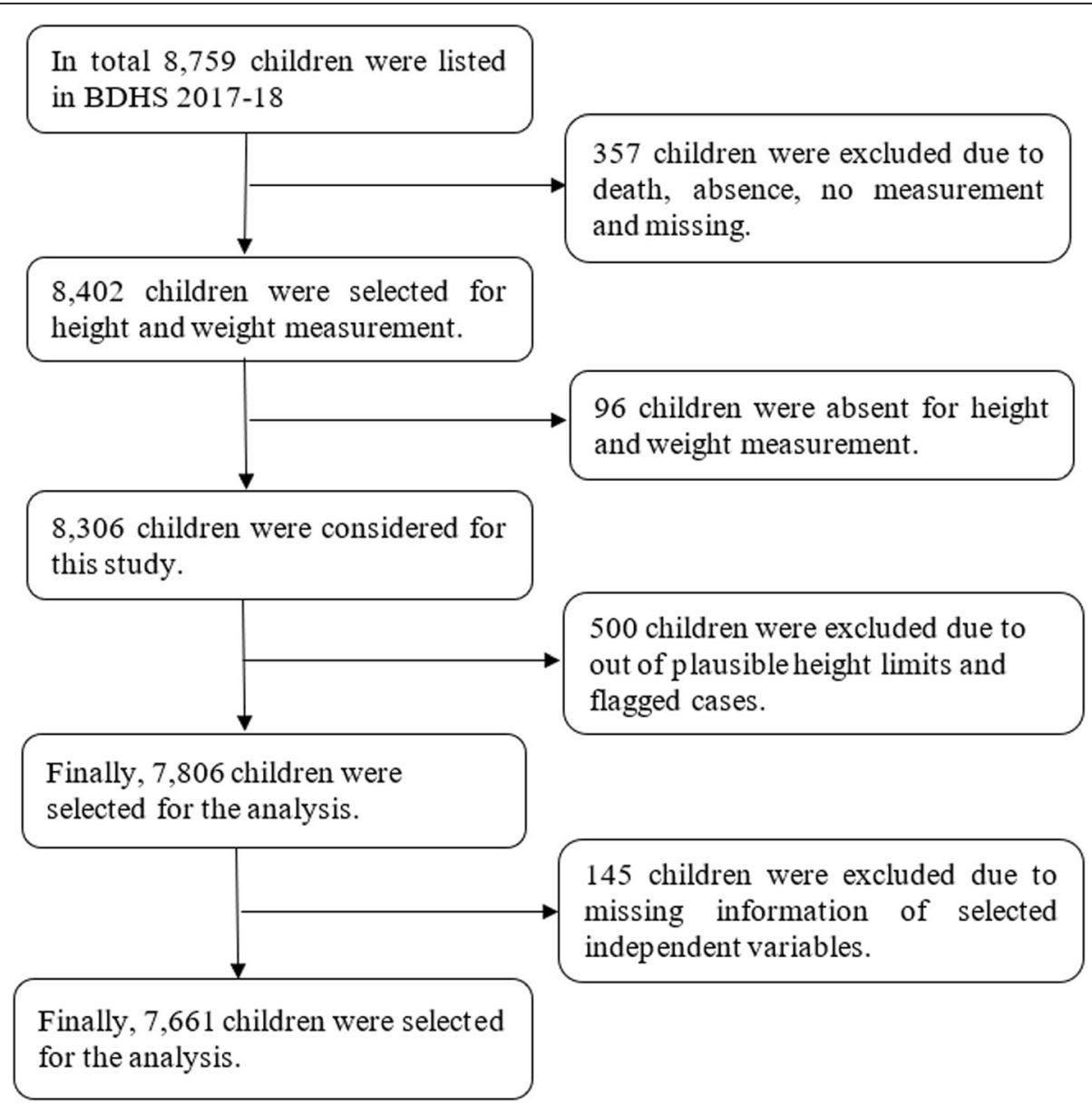

Fig. 1 Sample size selection 
Table 1 Classification of children with severe anthropometric failure

\begin{tabular}{|c|c|c|c|c|}
\hline Group name & Description & Severe Wasting & Severe Stunting & Severe Underweight \\
\hline A & No severe failure & No & No & No \\
\hline B & Severe wasting only & Yes & No & No \\
\hline C & Severe wasting and severe underweight & Yes & No & Yes \\
\hline $\mathrm{D}$ & Severe wasting, severe stunting and severe underweight & Yes & Yes & Yes \\
\hline E & Severe stunting and severe underweight & No & Yes & Yes \\
\hline $\mathrm{F}$ & Severe stunting only & No & Yes & No \\
\hline G & Severe underweight only & No & No & Yes \\
\hline
\end{tabular}

HAZ/LAZ <-3SD; WHZ/WLZ <-3SD; WAZ <-3SD) below the respective median of the World Health Organization (WHO) reference population, respectively [18]. Children without any anthropometric failure from $\mathrm{B}$ to $\mathrm{G}$, that is, with moderate undernutrition and/or normal children were categorized as 'no severe failure'.

\section{Independent variables}

Independent variables were selected based on the previously identified socioeconomic factors $[5,11,17,19,20]$. These variables include maternal age in years (15-19, 2024, 25-29, 30-34, 35-39, 240); parents' education (both parents uneducated, only father was uneducated when mother was educated, only mother was uneducated when father was educated, both parents educated); mother's current income-earning status (not working, currently working); underweight mother (if the Body Mass Index (BMI) is below $18.5 \mathrm{kgm}^{-2}$, then 'yes'; if $\mathrm{BMI} \geq 18.5$ $\mathrm{kgm}^{-2}$, then 'no'); mothers received antenatal care (no, yes); received postnatal care (no, yes); mother's attitudes toward wife-beating ('justified' if mothers agree that a husband is justified in hitting or beating his wife if she went out without telling him or/and neglected the children or/ and she ever argued with her partner or/and burned food or/and refused to have sex; otherwise 'not justified') [8]; decision-making autonomy ('participated', if women make decisions alone or jointly with their husband in all three of the following areas: (1) their own health care, (2) major household purchases, and (3) visits to their family or relatives; otherwise, 'not participated') [8]; religion (Islam, others); source of drinking water (improved, unimproved) [8], cooking fuel ['solid fuel' (including charcoal, wood, straw/shrubs/grass, agricultural crops, and animal dung), and 'clean fuel' (including electricity and LPG/natural gas/biogas)] [8]; mass media exposure (no, yes: exposure to either radio, television, newspapers, or magazines at least once a week), wealth index (integrating household asset ownership and access to drinking water and sanitation) [8]. Moreover, factors related to children, such as age, birth order, and birth weight status [21, 22], recent morbidity status (child had at least one morbid condition out of diarrhea, fever and cough in the two weeks preceding the survey) [8] were included. The detailed construction procedures of all independent variables were tabulated in the Additional Table 1.

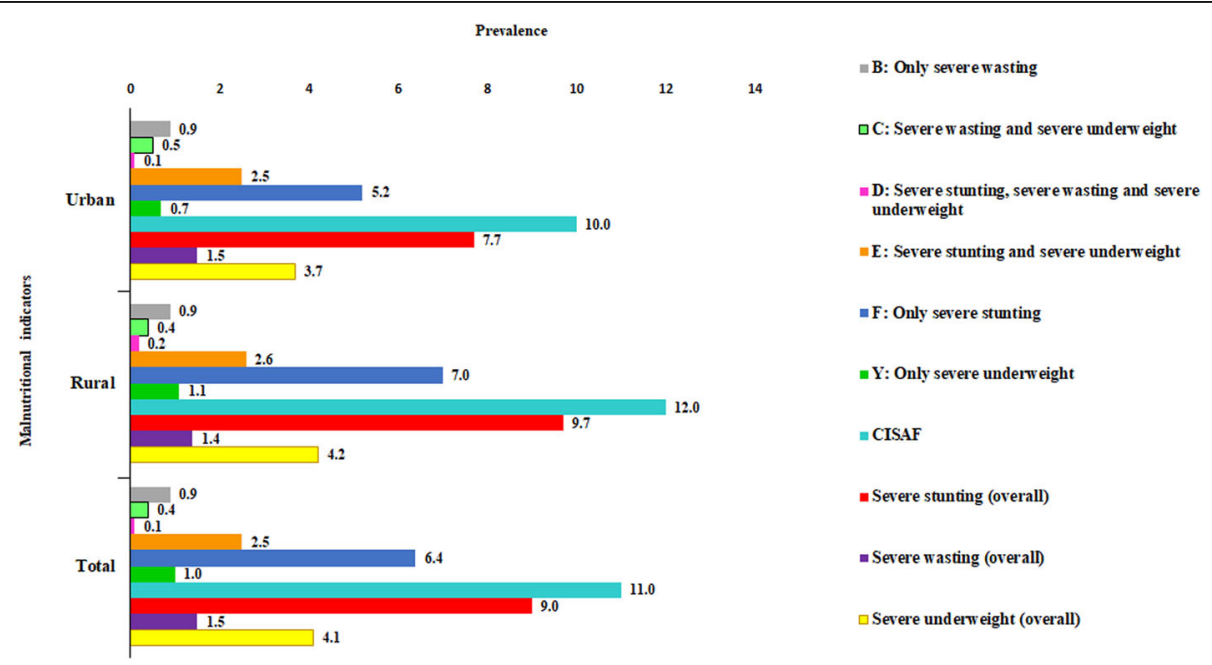

Fig. 2 Prevalence of malnutritional indicators 


\section{Statistical analysis}

Socioeconomic characteristics were analysed using descriptive statistics. Bivariate association was used to determine the proportional differences of variables between non-severe undernutrition and severe undernutrition group. The significance level for bivariate analysis was set at $P<0.10$ (2-tailed). Independent variables that were significant in bivariate analysis were simultaneously entered into the logistic regression models for the adjustment to identify the associated socioeconomic factors of severe undernutrition, measured by CISAF. Results were derived using a stepwise backward selection of regression and expressed by the adjusted odds ratio (AOR) and $95 \%$ confidence interval $(95 \% \mathrm{CI})$. The significance level for logistic regression analysis was set at $P<0.05$. To control the effect of the complex survey design, all the analyses of this study were performed using Stata's 'SVY' command. Variance Inflation Factor (VIF) was used to evaluate the possible collinearity. However, there was no multicollinearity problem among the variables. All statistical analyses were performed in Stata version 14.2 (StataCorp LP, College Station, Texas).

\section{Results}

\section{Background characteristics}

Of the 7661 under-5 children, about two-thirds lived in rural areas. Three-quarters of the mothers were aged below 30. In rural areas, families were comparatively least wealthy than the urban families (rural $54.3 \%$ vs. urban 19.4\%), and more mothers were underweight (rural $16 \%$ vs. urban $12.1 \%$ ). In approximately $82 \%$ of cases, both parents were educated, whereas nearly $4 \%$ of parents were uneducated, regardless of urban-rural settlement. Access to mass media was higher in urban areas (rural $56 \%$ vs. urban $79 \%$ ). Around $40 \%$ of the children were aged under 23 months. More than half of the children were not weighted during their birth. And nearly half of the children (47.2\%) had recently suffered from any morbidity condition (Table 2).

\section{Prevalence of severe undernutrition among the children under-5}

The overall prevalence of severe undernutrition measured by CISAF among the children under- 5 was $11.0 \%$ in Bangladesh (rural 11.5\% vs. urban 9.6\%) (Table 3). Figure 2 shows that the overall prevalences of severe stunting, severe wasting, and severe underweight were $9 \%, 1.5 \%$, and $4.1 \%$, respectively. The prevalence of severe stunting $(9.7 \%$ vs. $7.7 \%)$ and severe underweight $(4.2 \%$ vs. $3.7 \%)$ was higher in rural areas than in the urban (Fig. 2). In terms of regional prevalence, the Sylhet region reported the highest prevalence of severe under-5 child undernutrition in both rural (16.9\%) and urban areas (13\%) (Fig. 3).
From the bivariate association, in both urban and rural areas, children of uneducated parents (urban: $25.9 \%$ vs 8.4\%; rural: $21.5 \%$ vs $9.8 \%$ ), underweight mothers (urban: $15.4 \%$ vs $8.9 \%$; rural: $17.1 \%$ vs $10.5 \%$ ), poorest households (urban: $18.7 \%$ vs $6.4 \%$; rural: $16.1 \%$ vs $5.0 \%$ ), and with small birth weight (urban: $18.3 \%$ vs $6.6 \%$; rural:) reported a higher prevalence of severe under- 5 undernutrition than the children of educated parents, normal BMI mothers, richest households, and normal birth weight (Table 3).

\section{Socioeconomic factors associated with severe under-5 undernutrition}

The key associated factors for under- 5 undernutrition in urban areas were: children born with small birth weight (Adjusted Odds Ratio, AOR: 3.99, 95\% CI: 2.12, 7.52) vs. healthy weight; children of parents with no formal education (AOR: $2.34,95 \%$ CI: 1.27, 4.32) vs. children of educated parents; mothers did not receive postnatal care (AOR: $2.13,95 \%$ CI: 1.32, 3.44) vs. the recipient mothers; children's birth order $\geq 4$ (AOR: 1.75, 95\% CI: 1.97, 2.86) vs. children's first birth order; poorest children (AOR: $2.40,95 \%$ CI: $1.47,3.93)$ vs. the richest; and children of underweight mothers (AOR: 1.58, 95\% CI: 1.04, 2.40) vs. normal mothers (Table 4).

On the other hand, in the rural areas, children born with small birth weight (AOR: 2.84, 95\% CI: 1.88, 4.30) vs. healthy weight; children who lived in poorest households (AOR: 2.44, 95\% CI: 1.57, 3.78) vs. the richest households; children aged less than 36 months (for age group 24-35 months (AOR: 2.10, 95\% CI: 1.61, 2.74); for age group 12-23 months (AOR: 1.78, 95\% CI: 1.36, $2.33)$ ) vs. children of age group $0-11$ months; children of mothers with no formal education (when fathers are educated) (AOR: 2.15, 95\% CI: 1.46, 3.18) vs. children of educated parents, etc. socioeconomic factors showed a positive association with severe under- 5 undernutrition (Table 4).

\section{Discussion}

To the best of our knowledge, this is the first study in Bangladesh that used the updated version of the CIAF (i.e., the CISAF) to estimate the prevalence of severe under-5 undernutrition and its associated socioeconomic factors. Though the three most conventionally used stunting, wasting, and underweight indicators represent different physiological manifestations of undernutrition, they all share similar causal factors [23]. Individual assessments of stunting, wasting, and underweight underestimate the total burden of undernutrition. Since children may suffer from more than one type of undernutrition, only the data on prevalence may not provide a convincing estimate of the proportion of undernourished children in the population. Here the application of CIAS 
Table 2 Background characteristics of the children

\begin{tabular}{|c|c|c|c|}
\hline Characteristics & Total $(\%)^{\mathrm{e}}$ & Rural (\%) & Urban (\%) $^{\mathrm{e}}$ \\
\hline \multicolumn{4}{|l|}{ Maternal age (years) } \\
\hline $15-19$ & $938(12.2)$ & $657(13.0)$ & $281(10.8)$ \\
\hline $20-24$ & $2679(35.0)$ & $1818(36.0)$ & $861(33.0)$ \\
\hline $25-29$ & $2146(28.0)$ & $1395(27.6)$ & $751(28.8)$ \\
\hline $30-34$ & $1293(16.9)$ & $825(16.3)$ & $468(18.0)$ \\
\hline $34-39$ & $481(6.3)$ & $291(5.8)$ & $190(7.3)$ \\
\hline$\geq 40$ & $124(1.6)$ & $70(1.4)$ & $54(2.1)$ \\
\hline \multicolumn{4}{|l|}{ Parents' education } \\
\hline Both parents were uneducated & $294(3.8)$ & $208(4.1)$ & $86(3.3)$ \\
\hline Only father was uneducated & $865(11.3)$ & $654(12.9)$ & $211(8.1)$ \\
\hline Only mother was uneducated & $243(3.2)$ & $154(3.1)$ & $89(3.4)$ \\
\hline Both parents were educated & $6259(81.7)$ & $4040(79.9)$ & $2219(85.2)$ \\
\hline \multicolumn{4}{|l|}{ Mother's income-earning status } \\
\hline Not working & $4560(59.5)$ & $2788(55.1)$ & $1772(68.0)$ \\
\hline Currently working & $3101(40.5)$ & $2268(44.9)$ & $833(32.0)$ \\
\hline \multicolumn{4}{|l|}{ Underweight mother $\left(<18.5 \mathrm{~kg} / \mathrm{m}^{2}\right)$} \\
\hline No & $6535(85.3)$ & $4246(84.0)$ & 2289 (87.9) \\
\hline Yes & $1126(14.7)$ & $810(16.0)$ & $316(12.1)$ \\
\hline \multicolumn{4}{|c|}{ Mothers received antenatal care $(n=4540)$} \\
\hline No & $363(8.0)$ & $285(9.5)$ & $78(5.1)$ \\
\hline Yes & $4177(92.0)$ & $2727(90.5)$ & $1450(94.9)$ \\
\hline \multicolumn{4}{|c|}{ Mothers received postnatal care $(n=4535)$} \\
\hline No & $1509(33.3)$ & $970(32.2)$ & $539(35.4)$ \\
\hline Yes & $3026(66.7)$ & $2041(67.8)$ & $985(64.6)$ \\
\hline \multicolumn{4}{|c|}{ Mother's attitudes toward wife-beating } \\
\hline Not justified & $6274(81.9)$ & $4042(79.9)$ & $2232(85.7)$ \\
\hline Justified & $1387(18.1)$ & $1014(20.1)$ & $373(14.3)$ \\
\hline \multicolumn{4}{|c|}{ Mothers' decision-making autonomy ${ }^{\mathrm{a}}$} \\
\hline Not participated & $1083(14.1)$ & $786(15.5)$ & $297(11.4)$ \\
\hline Participated & $6578(85.9)$ & $4270(84.5)$ & $2308(71.9)$ \\
\hline \multicolumn{4}{|l|}{ Religion } \\
\hline Islam & $6997(91.3)$ & $4608(91.1)$ & $2389(91.7)$ \\
\hline Others & $664(8.7)$ & $448(8.9)$ & $216(8.3)$ \\
\hline \multicolumn{4}{|l|}{ Source of water } \\
\hline Improved & $6658(86.9)$ & $4332(85.7)$ & 2326 (89.3) \\
\hline Unimproved & $1003(13.1)$ & $724(14.3)$ & $279(10.7)$ \\
\hline \multicolumn{4}{|l|}{ Cooking fuel } \\
\hline Clean fuel & $2218(29.0)$ & 899 (17.8) & 1319 (50.6) \\
\hline Solid fuel & $5443(71.0)$ & $4157(82.2)$ & $1286(49.4)$ \\
\hline \multicolumn{4}{|l|}{ Type of toilet facility } \\
\hline Improved & $4359(56.9)$ & $2638(52.2)$ & $1721(66.1)$ \\
\hline Unimproved & $3302(43.1)$ & $2418(47.8)$ & $884(33.9)$ \\
\hline
\end{tabular}


Table 2 Background characteristics of the children (Continued)

\begin{tabular}{|c|c|c|c|}
\hline Characteristics & Total $(\%)^{\mathrm{e}}$ & Rural (\%) ${ }^{\mathrm{e}}$ & Urban (\%) $^{\mathrm{e}}$ \\
\hline \multicolumn{4}{|c|}{ Mass media exposure } \\
\hline No & $2771(36.1)$ & $2232(44.2)$ & $539(20.7)$ \\
\hline Yes & $4890(63.8)$ & $2824(55.8)$ & $2066(79.3)$ \\
\hline \multicolumn{4}{|l|}{ Wealth index } \\
\hline Poorest & $1708(22.3)$ & $1422(28.1)$ & $286(11.0)$ \\
\hline Poorer & $1545(20.2)$ & $1325(26.2)$ & $220(8.4)$ \\
\hline Middle & $1381(18.0)$ & $1024(20.2)$ & $357(13.7)$ \\
\hline Richer & $1533(20.0)$ & $827(16.4)$ & $706(27.1)$ \\
\hline Richest & $1494(19.5)$ & $458(9.1)$ & $1036(39.8)$ \\
\hline \multicolumn{4}{|l|}{ Children age } \\
\hline 0-11 months & $1673(21.8)$ & $1120(22.2)$ & $553(21.2)$ \\
\hline $12-23$ months & $1583(20.7)$ & $1058(20.9)$ & $525(20.1)$ \\
\hline 24-35 months & 1475 (19.3) & $973(19.2)$ & $502(19.3)$ \\
\hline $36-47$ months & $1417(18.5)$ & $939(18.6)$ & $478(18.4)$ \\
\hline $48-59$ months & $1513(19.7)$ & $966(19.1)$ & $547(21.0)$ \\
\hline \multicolumn{4}{|l|}{ Sex of child } \\
\hline Male & 3995 (51.2) & $2673(52.9)$ & $1322(50.7)$ \\
\hline Female & 3666 (48.8) & $2383(47.1)$ & $1283(49.3)$ \\
\hline \multicolumn{4}{|l|}{ Birth order } \\
\hline First & $2902(37.9)$ & $1848(36.6)$ & $1054(40.4)$ \\
\hline Second & $2507(32.7)$ & $1617(32.0)$ & $890(34.2)$ \\
\hline Third & $1297(16.9)$ & $893(17.7)$ & $404(15.5)$ \\
\hline Fourth and above & $955(12.5)$ & $698(13.8)$ & $257(9.9)$ \\
\hline \multicolumn{4}{|c|}{ Small birth weight ${ }^{\complement}(n=4735)$} \\
\hline No & 1815 (38.3) & $1036(32.9)$ & $779(49.2)$ \\
\hline Yes & $326(6.9)$ & $190(6.0)$ & $136(8.6)$ \\
\hline Not weighted & $2594(54.8)$ & $1927(61.1)$ & $667(42.2)$ \\
\hline \multicolumn{4}{|c|}{ Recent morbidity status ${ }^{d}$} \\
\hline No & $4043(52.8)$ & $2636(52.1)$ & $1407(54.0)$ \\
\hline Yes & $3618(47.2)$ & $2420(47.9)$ & $1198(46.0)$ \\
\hline Total & $7661(100.0)$ & $5056(66.0)$ & $2605(34.0)$ \\
\hline
\end{tabular}

adefined as women's decision making power relative to their male partners

bintegrating household asset ownership and access to drinking water and sanitation

'child's size and weight at birth based on a mother's perception

${ }^{d}$ child had at least one morbid condition out of diarrhea, fever and cough in the 2 weeks preceding the survey

epercentages were weighted

F identifies all malnourished children and provides a single estimate of the burden of severe undernutrition among the children under age 5 [15].

In this study, we used the aggregated CISAF measurement to investigate severe under- 5 child undernutrition in Bangladesh. More than one out of 10 children under5 (11\%) has been found to suffer from severe undernutrition. In a press release of UNICEF (2020), the prevalence of moderate to severe underweight and stunting was found to be about 23 and $28 \%$ in 2019, respectively [24]. Similarly, other organizations in Bangladesh (such as icddr,b, and Save the Children) working on child undernutrition have recently reported a similar prevalence rate of either severe stunting or underweight. But none of them used such a composite method to measure severe underweight, i.e., CISAF. So, the actual composite scenario of severe undernutrition among the under-5 children in Bangladesh has been overlooked. Severe stunting, underweight or child undernutrition in Bangladesh was investigated in different studies. Our research results are in line with these previous studies that rural under-5 children experience severe undernutrition 
Table 3 Proportion of severe under-5 child undernutrition in urban-rural context with respective to different socioeconomic characteristics

\begin{tabular}{|c|c|c|c|c|}
\hline \multirow[t]{2}{*}{ Characteristics } & \multicolumn{2}{|l|}{ Rural } & \multicolumn{2}{|l|}{ Urban } \\
\hline & Number & Prevalence $(95 \% \mathrm{Cl})$ & Number & Prevalence $(95 \% \mathrm{Cl})$ \\
\hline \multicolumn{5}{|l|}{ Maternal age (years) } \\
\hline $15-19$ & 85 & $11.5(9.2,14.2)$ & 36 & $13.2(8.9,19.1)$ \\
\hline $20-24$ & 201 & $10.5(8.9,12.3)$ & 77 & $9.5(6.9,12.9)$ \\
\hline $25-29$ & 176 & $12.3(10.4,14.5)$ & 68 & $7.9(6.0,10.4)$ \\
\hline $30-34$ & 103 & $12.4(9.9,15.5)$ & 41 & $8.6(5.8,12.6)$ \\
\hline $34-39$ & 35 & $11.0(7.9,15.1)$ & 84 & $13.9(8.8,21.1)$ \\
\hline \multirow[t]{2}{*}{$\geq 40$} & 9 & $11.8(6.1,21.7)$ & 7 & $9.8(3.8,23.1)$ \\
\hline & \multicolumn{2}{|c|}{$x^{2}=3.58, p=0.669$} & \multicolumn{2}{|c|}{$x^{2}=11.09, p=0.186$} \\
\hline \multicolumn{5}{|l|}{ Parents' education } \\
\hline Both parents uneducated & 42 & $21.5(15.7,28.8)$ & 23 & $25.9(17.2,36.9)$ \\
\hline Only father uneducated & 113 & $16.9(13.7,20.5)$ & 28 & $13.9(9.0,21.0)$ \\
\hline Only mother uneducated & 35 & $21.8(15.5,29.6)$ & 11 & $12.0(6.1,22.1)$ \\
\hline \multirow[t]{2}{*}{ Both parents educated } & 419 & $9.8(11.3,14.9)$ & 194 & $8.4(6.9,10.2)$ \\
\hline & \multicolumn{2}{|c|}{$x^{2}=64.76, p<0.001$} & \multicolumn{2}{|c|}{$x^{2}=37.89, p<0.001$} \\
\hline \multicolumn{5}{|c|}{ Mother's income-earning status } \\
\hline Not working & 321 & $10.7(9.2,12.3)$ & 166 & $8.8(7.1,10.8)$ \\
\hline \multirow[t]{2}{*}{ Currently working } & 288 & $12.6(11.1,14.2)$ & 90 & $11.4(8.9,14.6)$ \\
\hline & \multicolumn{2}{|c|}{$x^{2}=4.40, p=0.080$} & \multicolumn{2}{|c|}{$x^{2}=4.52, p=0.060$} \\
\hline \multicolumn{5}{|l|}{ Underweight mother } \\
\hline No & 468 & $10.5(9.4,11.8)$ & 201 & $8.9(7.3,10.7)$ \\
\hline \multirow[t]{2}{*}{ Yes } & 141 & $17.1(14.4,20.2)$ & 55 & $15.4(11.1,21.0)$ \\
\hline & \multicolumn{2}{|c|}{$x^{2}=26.99, p<0.001$} & \multicolumn{2}{|c|}{$x^{2}=12.99, p=0.001$} \\
\hline \multicolumn{5}{|c|}{ Mothers received antenatal care } \\
\hline No & 49 & $17.8(13.1,23.8)$ & 17 & $20.9(11.8,34.3)$ \\
\hline \multirow[t]{2}{*}{ Yes } & 325 & $11.2(9.9,12.5)$ & 147 & $9.9(8.1,12.0)$ \\
\hline & \multicolumn{2}{|c|}{$x^{2}=10.35, p=0.003$} & \multicolumn{2}{|c|}{$x^{2}=10.12, p=0.012$} \\
\hline \multicolumn{5}{|c|}{ Mothers received postnatal care } \\
\hline No & 92 & $9.5(7.6,11.9)$ & 40 & $5.9(4.0,8.8)$ \\
\hline \multirow[t]{2}{*}{ Yes } & 282 & $12.9(11.4,14.6)$ & 123 & $13.1(10.3,16.5)$ \\
\hline & & $p=0.015$ & & $p=0.001$ \\
\hline Mother's attitudes toward & & & & \\
\hline Not justified & 458 & $10.9(9.7,12.2)$ & 220 & $9.7(8.0,11.7)$ \\
\hline Justified & 151 & $13.9(11.5,16.6)$ & 36 & $9.1(5.8,14.0)$ \\
\hline & & $p=0.020$ & & $p=0.775$ \\
\hline Mothers' decision-making & & & & \\
\hline Not participated & 96 & $11.3(9.2,13.8)$ & 36 & $11.0(7.8,15.3)$ \\
\hline Participated & 513 & $11.5(10.3,12.9)$ & 220 & $9.4(7.7,11.4)$ \\
\hline & & $p=0.865$ & & $p=0.390$ \\
\hline Religion & & & & \\
\hline Islam & 557 & $11.5(10.4,12.8)$ & 236 & $9.7(8.0,11.7)$ \\
\hline Others & 52 & $11.0(8.5,14.2)$ & 20 & $8.5(4.6,15.1)$ \\
\hline & & $p=0.726$ & & $p=0.676$ \\
\hline
\end{tabular}


Table 3 Proportion of severe under-5 child undernutrition in urban-rural context with respective to different socioeconomic characteristics (Continued)

\begin{tabular}{|c|c|c|c|c|}
\hline \multirow[t]{2}{*}{ Characteristics } & \multicolumn{2}{|l|}{$\underline{\text { Rural }}$} & \multicolumn{2}{|l|}{ Urban } \\
\hline & Number & Prevalence $(95 \% \mathrm{Cl})$ & Number & Prevalence $(95 \% \mathrm{Cl})$ \\
\hline \multicolumn{5}{|c|}{ Source of drinking water } \\
\hline Improved & 532 & $11.8(10.6,13.1)$ & 229 & $9.6(8.0,11.6)$ \\
\hline \multirow[t]{2}{*}{ Unimproved } & 77 & $9.6(7.5,12.1)$ & 27 & $9.3(15.0,20.7)$ \\
\hline & \multicolumn{2}{|c|}{$x^{2}=3.03, p=0.102$} & \multicolumn{2}{|c|}{$x^{2}=0.03, p=0.862$} \\
\hline \multicolumn{5}{|l|}{ Cooking fuel } \\
\hline Clean fuel & 82 & $8.6(6.6,11.1)$ & 95 & $7.9(6.2,10.1)$ \\
\hline \multirow[t]{2}{*}{ Solid fuel } & 527 & $12.2(10.9,13.5)$ & 161 & $12.1(9.7,15.1)$ \\
\hline & \multicolumn{2}{|c|}{$x^{2}=9.98, p=0.011$} & \multicolumn{2}{|c|}{$x^{2}=12.99, p=0.005$} \\
\hline \multicolumn{5}{|l|}{ Type of toilet facility } \\
\hline Improved & 272 & $10.2(8.8,11.7)$ & 146 & $8.4(6.8,10.5)$ \\
\hline \multirow[t]{2}{*}{ Unimproved } & 337 & $13.0(11.5,14.6)$ & 110 & $12.0(8.9,16.0)$ \\
\hline & \multicolumn{2}{|c|}{$x^{2}=9.73, p=0.004$} & \multicolumn{2}{|c|}{$x^{2}=8.35, p=0.057$} \\
\hline \multicolumn{5}{|c|}{ Mass media exposure ${ }^{b}$} \\
\hline No & 328 & $14.6(12.7,16.7)$ & 82 & $15.4(10.8,21.4)$ \\
\hline \multirow[t]{2}{*}{ Yes } & 281 & $9.3(8.2,10.7)$ & 174 & $8.3(7.0,9.8)$ \\
\hline & \multicolumn{2}{|c|}{$x^{2}=33.16, p<0.001$} & \multicolumn{2}{|c|}{$x^{2}=23.01, p=0.008$} \\
\hline \multicolumn{5}{|l|}{ Wealth index } \\
\hline Poorest & 234 & $16.1(14.0,18.4)$ & 50 & $18.7(11.7,28.7)$ \\
\hline Poorer & 178 & $12.8(10.9,15.1)$ & 40 & $16.1(11.6,22.1)$ \\
\hline Middle & 98 & $9.2(7.3,11.6)$ & 41 & $10.6(7.2,15.4)$ \\
\hline Richer & 75 & $8.9(6.8,11.6)$ & 63 & $9.7(7.1,13.1)$ \\
\hline \multirow[t]{2}{*}{ Richest } & 24 & $5.0(3.1,8.1)$ & 62 & $6.4(4.9,8.3)$ \\
\hline & \multicolumn{2}{|c|}{$x^{2}=60.34, p<0.001$} & \multicolumn{2}{|c|}{$x^{2}=44.82, p=<0.001$} \\
\hline \multicolumn{5}{|l|}{ Children age } \\
\hline 0-11 months & 95 & $8.1(6.6,10.0)$ & 53 & $7.5(5.0,11.0)$ \\
\hline $12-23$ months & 153 & $13.4(11.3,15.8)$ & 56 & $10.4(7.6,14.1)$ \\
\hline 24-35 months & 155 & $15.2(12.8,17.9)$ & 62 & $14.2(10.8,18.6)$ \\
\hline 36-47 months & 102 & $10.8(8.6,13.5)$ & 41 & $8.2(5.6,12.0)$ \\
\hline \multirow[t]{2}{*}{ 48-59 months } & 104 & $10.1(8.1,12.6)$ & 44 & $7.9(5.5,11.3)$ \\
\hline & \multicolumn{2}{|c|}{$x^{2}=31.49, p<0.001$} & & $p=0.013$ \\
\hline Sex of child & & & & \\
\hline Male & 326 & $11.4(10.1,12.9)$ & 137 & $10.5(8.5,12.8)$ \\
\hline Female & 283 & $11.6(10.0,13.4)$ & 119 & $8.7(6.8,11.1)$ \\
\hline & & $p=0.871$ & & $p=0.159$ \\
\hline Birth order & & & & \\
\hline First & 197 & $10.1(8.6,11.8)$ & 84 & $8.3(6.4,10.9)$ \\
\hline Second & 184 & $10.7(9.1,12.4)$ & 83 & $8.7(6.5,11.7)$ \\
\hline Third & 114 & $12.3(10.1,14.8)$ & 41 & $9.3(6.5,13.0)$ \\
\hline Fourth and above & 114 & $16.2(12.8,20.3)$ & 48 & $18.9(14.1,24.8)$ \\
\hline & & $p=0.001$ & & $p=0.002$ \\
\hline
\end{tabular}


Table 3 Proportion of severe under-5 child undernutrition in urban-rural context with respective to different socioeconomic characteristics (Continued)

\begin{tabular}{|c|c|c|c|c|}
\hline \multirow[t]{2}{*}{ Characteristics } & \multicolumn{2}{|l|}{ Rural } & \multicolumn{2}{|l|}{ Urban } \\
\hline & Number & Prevalence $(95 \% \mathrm{Cl})$ & Number & Prevalence $(95 \% \mathrm{Cl})$ \\
\hline \multicolumn{5}{|c|}{ Small birth weight $^{d}$} \\
\hline No & 74 & $6.6(5.2,8.4)$ & 47 & $6.6(4.8,9.0)$ \\
\hline \multirow[t]{2}{*}{ Yes } & 32 & $18.3(12.8,25.3)$ & 29 & $18.3(11.8,27.1)$ \\
\hline & \multicolumn{2}{|c|}{$x^{2}=48.54, p<0.001$} & \multicolumn{2}{|c|}{$x^{2}=29.06, p=0.003$} \\
\hline \multicolumn{5}{|c|}{ Recent morbidity status ${ }^{\mathrm{e}}$} \\
\hline No & 112 & $11.2(9.8,12.7)$ & 147 & $10.4(8.4,12.9)$ \\
\hline \multirow[t]{2}{*}{ Yes } & 297 & $11.8(10.4,13.5)$ & 109 & $8.7(6.7,11.1)$ \\
\hline & \multicolumn{2}{|c|}{$x^{2}=0.53, p=0.495$} & \multicolumn{2}{|c|}{$x^{2}=2.32, p=0.206$} \\
\hline Total & 609 & $11.5(10.4,12.7)$ & 256 & $9.6(8.0,11.5)$ \\
\hline
\end{tabular}

adefined as women's decision-making power relative to their male partners

bexposure to either radio, television, newspapers, or magazines at least once a week

integrating household asset ownership and access to drinking water and sanitation

dchild's size and weight at birth based on a mother's perception

${ }^{e}$ child had at least one morbid condition out of diarrhea, fever and cough in the 2 weeks preceding the survey

$p$ values are obtained from Chi-square estimation

than the urban ones [25-28]. But the composite index for measuring severe undernutrition, that is CISAF was not applied in those studies.

On the contrary, according to a few studies, severe under- 5 undernutrition was more prevalent in the urban areas of Bangladesh [29, 30]. It is noteworthy that these studies $[29,30]$ used the data of the last two decades. There has been a significant change in the urban areas of Bangladesh for the last few years, such as universal access to primary education, improving the household socioeconomic status, adequate maternal healthcare utilization, improvement of transportation facilities, and awareness about nutrition, which ultimately improved the overall nutritional status in the urban areas [27-29]. Compared to this, rural areas did not get the touch of

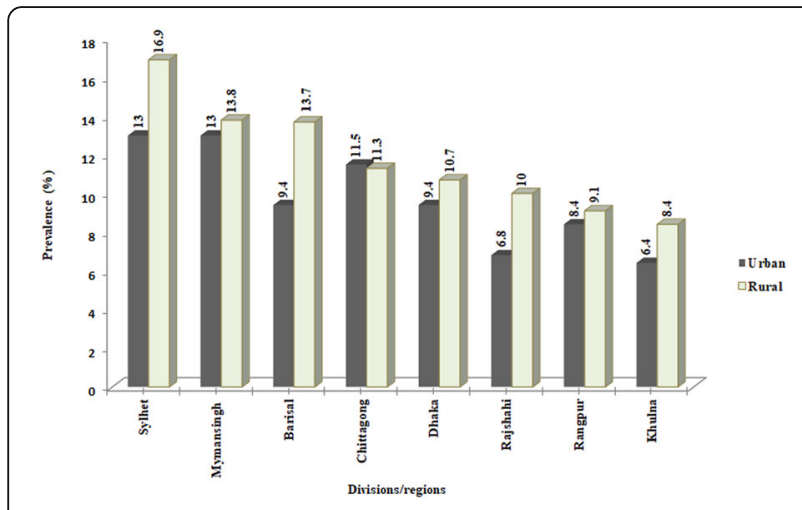

Fig. 3 Regional prevalence in urban-rural context such extensive development in Bangladesh. It might be one of the plausible explanations behind such a higher prevalence of severe under- 5 undernutrition among the rural children of Bangladesh.

The prevalence of severe under- 5 child undernutrition was more dominant among the children of uneducated parents. One in four children of the uneducated parents experienced severe undernutrition regardless of being born in rural or urban areas. Further, low birth weight children had a greater odd of being severely malnourished irrespective of the rural or urban context. For example, in our study, children with low birth weight were more likely to experience severe undernutrition (3.99 times the odds) living in the urban areas than rural children with low birth weight (2.84 times the odds). Also, one-fifth of the under- 5 children born in rural and urban areas with a small birth weight experienced severe undernutrition. These findings were matched with the previous studies of Pakistan, Nepal, Malawi, Mexico, and Iran, which reported that children born with small birth weight were more likely to experience different types of undernutrition [19, 31-34]. Generally, children born with a low birth weight gain inadequate amounts of height and weight [35]. Thus, they may remain shorter and lighter, and might suffer from severe undernutrition without adequate nutritional support. Additionally, children with small birth weights are often born to households with low socioeconomic status and mothers with poor health conditions [36]. Due to the irregular distribution of food for children in poverty-stricken households, and the knowledge gap of parents/caregivers for adequate micronutrient supplementation, children can 
Table 4 Association between socioeconomic characteristics and under-5 severe child undernutrition in rural-urban context

\begin{tabular}{|c|c|c|c|c|}
\hline \multirow{2}{*}{$\begin{array}{l}\text { Socioeconomic } \\
\text { characteristics }\end{array}$} & \multicolumn{2}{|l|}{ Rural } & \multicolumn{2}{|l|}{ Urban } \\
\hline & Adjusted Odds Ratio (95\% Cl) & $P$ values & Adjusted Odds Ratio (95\% Cl) & $P$ values \\
\hline \multicolumn{5}{|l|}{ Parents' education $^{a}$} \\
\hline Both parents uneducated & $1.92(1.35-2.73)$ & $<0.001$ & $2.34(1.27-4.32)$ & 0.006 \\
\hline Only father uneducated & $1.54(1.23-1.94)$ & $<0.001$ & $1.42(0.86-2.36)$ & 0.170 \\
\hline Only mother uneducated & $2.15(1.46-3.18)$ & $<0.001$ & $1.08(0.51-2.29)$ & 0.843 \\
\hline Both parents educated (RC) & 1.00 & & 1.00 & \\
\hline \multicolumn{5}{|c|}{ Underweight mother $(<18.5 \mathrm{~kg} / \mathrm{m} 2)^{a}$} \\
\hline No $(\mathrm{RC})$ & 1.00 & & 1.00 & \\
\hline Yes & $1.50(1.21-1.84)$ & $<0.001$ & $1.58(1.04-2.40)$ & 0.033 \\
\hline
\end{tabular}

Mothers received antenatal care ${ }^{b}$

$\begin{array}{ll}\text { No } & 1.28(1.00-1.64) \\ \text { Yes (RC) } & 1.00\end{array}$

0.050

Mothers received postnatal care ${ }^{b}$

No

Yes (RC)

Mother's attitudes toward wife-beating ${ }^{\text {a }}$

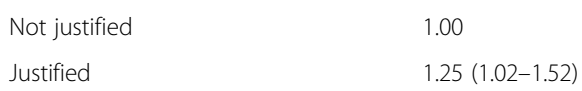

\subsection{0}

$.25(1.02-1.52)$

Mass media exposure ${ }^{a, c}$

No

Yes (RC)

Wealth index ${ }^{\text {a,d }}$

$\begin{array}{ll}\text { Poorest } & 2.44(1.57-3.78) \\ \text { Poorer } & 2.20(1.43-3.38) \\ \text { Middle } & 1.68(1.09-2.61) \\ \text { Richer } & 1.80(1.15-2.82) \\ \text { Richest (RC) } & 1.00\end{array}$

\section{Children age ${ }^{a}$}

$\begin{array}{ll}0-11 \text { months }(\mathrm{RC}) & 1.00 \\ 12-23 \text { months } & 1.78(1.36-2.33) \\ 24-35 \text { months } & 2.10(1.61-2.74) \\ 36-47 \text { months } & 1.37(1.03-1.83) \\ 48-59 \text { months } & 1.21(0.90-1.61)\end{array}$

Birth order ${ }^{a}$

First (RC)

Second

Third

Fourth and above

0.028

0.018

$<0.001$

$2.40(1.47-3.93)$

$<0.001$

$<0.001$

0.020

0.011

$2.35(1.34-4.11)$

0.003

$1.49(0.90-2.46)$

$1.36(0.91-2.02)$

0.122

0.132

1.00

$\begin{array}{lll}<0.001 & 1.00 & \\ <0.001 & 1.46(0.89-2.39) & 0.131 \\ 0.030 & 2.19(1.37-3.51) & 0.001 \\ 0.202 & 1.16(0.69-1.96) & 0.577 \\ & 1.08(0.64-1.83) & 0.765\end{array}$

1.00

$1.07(0.74-1.54) \quad 0.728$

$1.05(0.65-1.69) \quad 0.833$

$1.75(1.07-2.86)$

Small birth weight ${ }^{\text {b,e }}$

$\begin{array}{ll}\text { No }(\mathrm{RC}) & 1.00 \\ \text { Yes } & 2.84(1.88-4.30)\end{array}$

$\mathrm{RC}$, reference category

a model adjusted with all variables found significant in the bivariate analysis except mothers received antenatal care, mothers received postnatal care, and small birth weight

b model adjusted with all variables found significant in the bivariate analysis including mothers received antenatal care, mothers received postnatal care and small birth weight

c exposure to either radio, television, newspapers, or magazines at least once a week

d integrating household asset ownership and access to drinking water and sanitation

e child's weight at birth based on measuring and mother's perception

Results are derived using stepwise backward selection of regression 
be deprived of nutritious food intake and eventually suffer from acute undernutrition [12, 14]. Again, parental illiteracy is frequently connected with low birth weight and other variables, such as inadequate maternal healthcare access and child caregiving. All these interlinked factors are substantially responsible for the poor nutritional outcomes of mothers and the low birth weight among the children [37, 38].

Poor household status has been found as one of the strongly associated factors for severe undernutrition among under-5 children in several studies $[5,39,40]$, which is matched with our findings. Nearly one-fifth of the children who lived in the least wealthy households experienced severe under- 5 undernutrition, regardless of the urban-rural context of this study. The odds of severe under-5 undernutrition were 2.44 times higher for children living in rural areas and 2.40 folds higher for urban children from the poorest wealth index. Poor parents often cannot afford a minimum diet and proper postnatal care for their children [41]. Other plausible explanations, such as increased clustering of urban poor in slums with limited access to public health and nutrition services and amenities, high population density, poor quality drinking water, inadequate sanitation facilities, are also blameworthy for the higher prevalence of severeundernutrition among the under- 5 children of the poor households [42].

In this study, uneducated parents were one of the strongly associated factors of severe child undernutrition, especially in rural areas. Educated parents were found to be significantly associated with better nutritional conditions during pregnancy and after birth and had been an indirect predictor of better child health throughout life [43, 44]. According to the studies from Bangladesh [43] and Pakistan [44], the likelihood of child undernutrition increased when their parents were uneducated, which is consistent with our study findings in rural areas. Again, maternal education could be an important determinant of child survival in rural areas since the child mortality and morbidity rates are usually high [45]. Like our study finding, maternal lower educational status in rural areas increases the odds of severe child undernutrition. Hence, a greater focus on parental education facilities is required irrespective of geographical regions to accelerate the nationwide improvements in child nutrition status.

Children's birth order $\geq 4$ were 1.75 times more likely to experience severely malnourished living in urban areas. Studies from Bangladesh and India showed that children in higher birth order were more likely to be severely malnourished in Bangladesh's urban areas [25] but in rural areas of India [46]. Previous studies from Bangladesh [5], Congo [47], and Ethiopia [48] also reported that children with higher birth order were more likely to experience undernutrition regardless of urbanrural context. When the number of children increases, household members get competitive for foods, and the equality of providing necessary foods could not be maintained for all children [49]. The risk of severe undernutrition is usually high in older children (i.e., age 4 to 5 years) in Nepal, Pakistan, Ethiopia, and Congo [17, 48, $50,51]$.

In comparison, severe undernutrition is high in younger (age 1 to 2 years) children in India [52], indicating this problem's complex nature. We found that toddlers (age 2 to 3 years) living in rural areas had higher odds of severe under- 5 undernutrition than toddlers living in urban areas. A similar level of provision of health and nutritional care available for urban children might be the reason for the insignificant association between children's age and severe undernutrition. At 6-36 months, inappropriate feeding behaviors and other factors (e.g., infection and food shortage) may be responsible for onethird of undernutrition cases, depending on population, place, time, and season [53]. In addition, lack of attention in rural areas (urban-rural disparities) in receiving complementary feeding, access to health services, preventive and curative interventions influence nutrition outcomes [54].

Severeundernutrition is a multifaceted, complex phenomenon, involving many immediate causes (e.g., poor diet habits, diarrhea, ages of breastfeeding children) and underlying causes (e.g., income inequality, food insecurity, access to safe water, environmental hygiene) [55, 56]. The risk difference between most affluent and poorest was higher among children in rural areas than in urban areas, indicating a greater rich-poor gap. Such socioeconomic inequality can be reduced by increasing income-generating activities driven by public and private entities and introducing different microfinance programs. These endeavors need to be aimed at deprived and vulnerable individuals and ensure their participation with a standard wage structure under the national nutritional security system. In Ethiopia, improved per capita household income increased available funds for food expenditure and basic health care needs and developed children's nutritional status [57]. Moreover, universal education and standard health care should be available and accessible to all women, especially in rural and remote areas. Improving access to community-based education and standard health care to mother include conferring many benefits from improved caregiver practices; enhancing health and environmental knowledge; increasing educated and skilled workforce; living in better neighborhoods, reducing gender-based violence, child marriage, and early childbearing; and reducing maternal death rates in terms of improved maternal and child nutritional status [58]. 


\section{Limitations}

The BDHS 2017-18 data used for this study was one of Bangladesh's largest nationally representative samples. The stability of the data set allows changes over time to be monitored with some confidence. However, there were some limitations too for this study. First, we could not use the infant and young children feeding data to the models because the data available in the BDHS were only for the children aged 6 to 23 months. But our study considered children under age 5 . So, there would be too many missing variables, and this study would be limited only to children under age 2. Further, data on potential confounders like diet, food insecurity, and parents smoking behavior were unavailable. Secondly, the crosssectional data was insufficient to establish a causal relationship, consequently limiting the findings' applicability. Thirdly, the BDHS data were collected retrospectively and self-reported, thus subject to underreporting, information bias, and recall bias. However, data were collected using validated tools and standard procedures. Using seven nutritional status measurements, CISAF provided a credible estimate of the overall proportion of severe under-5 child undernutrition and the complex interplay between individual, community, public policy, and environment level associated factors.

\section{Conclusion}

The overall prevalence of severe under- 5 child undernutrition was greater than conventional indicators. One in ten children living in both rural and urban areas experience severe undernutrition. Rural under-5 children were experiencing more severe undernutrition than the urban ones. Children of uneducated parents, underweight mothers, living in the poorest households, and with small birth weight experienced severe undernutrition regardless of urban-rural setting. Rural areas were identified as a greater rich-poor gap. The findings of this study imply that more research should be conducted from different aspects using such composite index (likeCISAF) to depict the comprehensive scenario of severe undernutrition among the under-5 children in Bangladesh. From a policy-making perspective, only the educational attainment and access to health and nutritional care may not be enough to reduce the burden of severe under-5 child undernutrition in rural settings due to the complex interplay between the socioeconomic and cultural factors. Bangladesh needs to focus on household wealth generation by introducing intervening microfinance programs, improving maternal health through proper maternal healthcare utilization, and different intervention programs on vulnerable groups, including the children from the poorest socioeconomic strata or children in the urban areas. Additionally, strengthening the role of parents through awareness programs and yard meetings to improve their knowledge in different aspects like- family planning, reduction of fertility, child nutrition, and maternal care. Furthermore, to achieve the SDGs by 2030, a targeted multi-sectoral healthcare program is required to reduce the prevalence of short-statured children at an early age, to advance knowledge on complementary foods for young children, and to reinforce family planning programs aimed at increasing the age at birth and decreasing higher-order births.

\section{Abbreviations}

AOR: Adjusted Odds Ratio; BDHS: Bangladesh Demographic Health Survey; Cl: Confidence Interval; CIAF: Composite Index of Anthropometric Failure; CISAF: Composite Index of Severe Anthropometric Failure; EA: Enumeration Area; LPG: Liquid Petroleum Gas; PSU: Primary Sampling Unit;

SDG: Sustainable Development Goal; WHO: World Health Organization

\section{Supplementary Information}

The online version contains supplementary material available at https://doi. org/10.1186/s12889-021-12038-3.

\section{Additional file 1}

\section{Acknowledgments}

Not Applicable.

\section{Authors' contributions}

MRKC conceptualized the basic idea for the study, performed the statistical analysis together with AIA. MRKC and AIA prepared data for analysis. AIA, MRKC, MK prepared the first draft of the manuscript. HTAK, MNIM, and NKPP critically revised the manuscript for intellectual content. All authors have reviewed and approved the final manuscript.

\section{Funding}

This research received no specific grant from any institutions. Open Access funding provided by Karolinska Institute.

\section{Availability of data and materials}

The data underlying the results presented in the study are publicly accessible and available from the DHS website (https://dhsprogram.com/ data/available-datasets.cfm). The name of the dataset is Bangladesh Demographic and Health Survey (BDHS)

\section{Declarations}

Ethics approval and consent to participate No ethical approval was needed for this study since it was based on secondary analysis of the data obtained from Bangladesh Demographic and Health Survey (BDHSs) between 2007 and 2018. The BDHSs were reviewed and approved by the ICF Macro Institutional Review Board (USA), which complies with all the requirements of 45 CFR 46 "Protection of Human Subjects". The Bangladesh DHS was also reviewed and approved by the National Research Ethics Committee of the Bangladesh Medical Research Council (Dhaka, Bangladesh). The survey ensured international ethical standards of confidentiality, anonymity, and informed consent. However, a request to access datasets from the measure DHS website is made and the websites have allowed the same before analyses are made.

All methods were carried out in accordance with relevant guidelines and regulations.

Consent for publication

Not applicable.

Competing interests

The authors declare that they have no competing interests. 


\section{Author details}

'Department of Public Health and Informatics, Bangabandhu Sheikh Mujib Medical University (BSMMU), Shahbag, Dhaka, Bangladesh. ${ }^{2}$ Department of Research and Evaluation, SAJIDA Foundation, Gulshan-1, Dhaka, Bangladesh. ${ }^{3}$ Department of Public Health, First Capital University of Bangladesh, Chuadanga, Bangladesh. ${ }^{4}$ College of Nursing, Midwifery and Healthcare, University of West London, London, UK. ${ }^{5}$ Department of Population Science and Human Resource Development, University of Rajshahi, Rajshahi, Bangladesh. ${ }^{6}$ Nuffield Department of Orthopaedics, Rheumatology, and Musculoskeletal Sciences, University of Oxford, Oxford, UK. ${ }^{7}$ Unit of Physiotherapy, Department of Health, Medicine and Caring Sciences (HMV), Linköping University, Linköping, Sweden. ${ }^{8}$ Unit of Occupational Medicine, Institute of Environmental Medicine | Karolinska Institutet, Solnavägen 4, Torsplan floor 10, 11365 Stockholm, Sweden.

Received: 7 April 2021 Accepted: 15 October 2021

Published online: 23 November 2021

\section{References}

1. Pelletier DL, Frongillo EA. Changes in child survival are strongly associated with changes in malnutrition in developing countries. J Nutr. 2003;133(1): 107-19. https://doi.org/10.1093/jn/133.1.107.

2. Black RE, Allen LH, Bhutta ZA, Caulfield LE, De Onis M, Ezzati M, et al. Maternal and child undernutrition: global and regional exposures and health consequences. Lancet. 2008;371(9608):243-60. https://doi.org/10.101 6/S0140-6736(07)61690-0.

3. UNICEF, WHO, World bank group. Malnutrition rates remain alarming: stunting is declining too slowly while overweight continues to rise 2016. https://data.unicef.org/wp-content/uploads/2016/09/UNICEF-Joint-Ma Inutrition-brochure.pdf. .

4. Akhtar S. Malnutrition in South Asia - a critical reappraisal. Crit Rev Food Sci Nutr. 2016;56(14):2320-30. https://doi.org/10.1080/10408398.2013.832143.

5. Chowdhury MRK, Khan HTA, Mondal MNI, Kabir R. Sociodemographic risk factors for severe malnutrition in children aged under five among various birth cohorts in Bangladesh. J Biosoc Sci. 2021;53(4):590-605. https://doi. org/10.1017/S0021932020000425.

6. Black RE, Victora CG, Walker SP, Bhutta ZA, Christian P, de Onis M, et al. Maternal and child undernutrition and overweight in low-income and middle-income countries. Lancet. 2013;382(9890):427-51. https://doi.org/1 0.1016/50140-6736(13)60937-X.

7. Khan REA, Raza MA. Nutritional status of children in Bangladesh: measuring composite index of anthropometric failure (CIAF) and its determinants; 2014

8. National Institute of Population Research and Training - NIPORT, Ministry of Health and Family Welfare, ICF. Bangladesh Demographic and Health Survey 2017-18. Dhaka, Bangladesh: NIPORT/ICF; 2020. https://www. dhsprogram.com/pubs/pdf/FR344/FR344.pdf.

9. United Nations. The Sustainable Development Goals 2016. eSocialSciences; 2016. https://sustainabledevelopment.un.org/content/documents/2125203 0\%20Agenda\%20for\%20Sustainable\%20Development\%20web.pdf.

10. World Health Organization. A conceptual framework for action on the social determinants of health. Debates, Policy \& Practice, Case Studies. Geneva, Switzerland: World Health Organization; 2010.

11. Islam MR, Rahman MS, Rahman MM, Nomura S, de Silva A, Lanerolle P, et al. Reducing childhood malnutrition in Bangladesh: the importance of addressing socioeconomic inequalities. Public Health Nutr. 2020;23(1):72-82. https://doi.org/10.1017/S136898001900140X.

12. Madhusudhan K, Rajeev PK, Shireesha A, Ushashree GV. Study of risk factors of severe acute malnutrition (SAM) in children 6 months to 5 years of age and evaluation of effect of micronutrient supplementation (WHO protocol) on serum zinc and magnesium levels: a case control study. Int. J. Contemp. Pediatr. 2017:4:1198-205.

13. Kamal S. Socio-economic determinants of severe and moderate stunting among under-five children of rural Bangladesh. Malays J Nutr. 2011;17:105-18.

14. Nandy S, Irving M, Gordon D, Subramanian S, Smith GD. Poverty, child undernutrition and morbidity: new evidence from India. Bull World Health Organ 2005;83:210-216, 3, DOI: /S0042-96862005000300014.

15. Vollmer S, Harttgen K, Kupka R, Subramanian S. Levels and trends of childhood undernutrition by wealth and education according to a composite index of anthropometric failure: evidence from 146 demographic and health surveys from 39 countries. BMJ Glob Health. 2017;2(2):e000206. https://doi.org/10.1136/bmjgh-2016-000206.
16. Chowdhury MRK, Khan HTA, Mondal MNI. Differences in the sociodemographic determinants of undernutrition in children aged $<5$ years in urban and rural areas of Bangladesh measured by the composite index of anthropometric failure. Public Health. 2021;198:37-43. https://doi. org/10.1016/j.puhe.2021.06.027.

17. Anik Al, Rahman MM, Rahman MM, Tareque MI, Khan MN, Alam MM. Double burden of malnutrition at household level: a comparative study among Bangladesh, Nepal, Pakistan, and Myanmar. PLoS One. 2019;14(8): e0221274. https://doi.org/10.1371/journal.pone.0221274.

18. World Health Organization. WHO child growth standards: length/height-forage, weight-for-age, weight-for-length, weight-for-height and body mass index-for-age: methods and development. World Health Organization; 2006.

19. Tiwari $R$, Ausman LM, Agho KE. Determinants of stunting and severe stunting among under-fives: evidence from the 2011 Nepal demographic and health survey. BMC Pediatr. 2014;14(1):1-15. https://doi.org/10.1186/14 71-2431-14-239.

20. Akombi BJ, Agho KE, Hall JJ, Merom D, Astell-Burt T, Renzaho AM. Stunting and severe stunting among children under-5 years in Nigeria: a multilevel analysis. BMC Pediatr. 2017;17(1):1-16. https://doi.org/10.1186/s12887-0160770-z.

21. Sreeramareddy CT, Shidhaye RR, Sathiakumar N. Association between biomass fuel use and maternal report of child size at birth-an analysis of 2005-06 India demographic health survey data. BMC Public Health. 2011; 11(1):1-10. https://doi.org/10.1186/1471-2458-11-403.

22. Haque SR, Tisha S, Huq N. Poor birth size a badge of low birth weight accompanying less antenatal care in Bangladesh with substantial divisional variation: evidence from BDHS-2011. Birth. 2015;1:33-8.

23. Khara T, Dolan C. Technical Briefing Paper: Associations Between Wasting and Stunting, Policy, Programming and Research Implications. The relationship between wasting and stunting: policy, programming and research implications. 2014.

24. UNICEF, Bangladesh Bureau of Statistics. Bangladesh sees sharp decline in child malnutrition, while violent disciplining of children rises, new survey reveals. Bangladesh; 2020. https://www.unicef.org/bangladesh/en/pressreleases/bangladesh-sees-sharp-decline-child-malnutrition-while-violentdisciplining-children. Accessed 15 Sep 2021.

25. Akram R, Sultana M, Ali N, Sheikh N, Sarker AR. Prevalence and determinants of stunting among preschool children and its urban-rural disparities in Bangladesh. Food Nutr Bull. 2018;39(4):521-35. https://doi.org/10.1177/03 79572118794770.

26. Hasan M, Hoque M, Hossain M, Mollah A, Islam M, Ahsan M, et al. Nutritional status among primary school children of Mymensingh. MMJ. 2013;22(2):267-74.

27. Rahman A, Rahman MS. Rural-urban differentials of childhood malnutrition in Bangladesh. Int. J. Child Health Nutr. 2019;8(1):35-42. https://doi.org/10. 6000/1929-4247.2019.08.01.5.

28. Rahman MS, Rahman MA, Maniruzzaman M, Howlader MH. Prevalence of undernutrition in Bangladeshi children. J Biosoc Sci. 2020;52(4):596-609. https://doi.org/10.1017/S0021932019000683.

29. Das SK, Chisti MJ, Malek MA, Das J, Salam MA, Ahmed T, et al. Changing childhood malnutrition in Bangladesh: trends over the last two decades in urban-rural differentials (1993-2012). Public Health Nutr. 2015;18(10):171827. https://doi.org/10.1017/S136898001500004X.

30. Saha UR, Chattapadhayay A, Richardus JH. Trends, prevalence and determinants of childhood chronic undernutrition in regional divisions of Bangladesh: evidence from demographic health surveys, 2011 and 2014. PLoS One. 2019;14(8):e0220062. https://doi.org/10.1371/journal.pone.0220062.

31. Saleemi M, Ashraf R, Mellander L, Zaman S. Determinants of stunting at 6, 12, 24 and 60 months and postnatal linear growth in Pakistani children. Acta Paediatr. 2001;90(11):1304-8. https://doi.org/10.1080/080352501317130371.

32. Ntenda PAM. Association of low birth weight with undernutrition in preschool-aged children in Malawi. Nutr J. 2019;18(1):1-15. https://doi.org/1 0.1186/s12937-019-0477-8.

33. Jahanihashemi H, Noroozi M, Zavoshy R, Afkhamrezaei A, Jalilolghadr S, Esmailzadehha N. Malnutrition and birth related determinants among children in Qazvin, Iran. Eur J Public Health. 2017;27(3):559-62. https://doi. org/10.1093/eurpub/ckx043.

34. Varela-Silva MI, Azcorra H, Dickinson F, Bogin B, Frisancho A. Influence of maternal stature, pregnancy age, and infant birth weight on growth during childhood in Yucatan, Mexico: a test of the intergenerational effects hypothesis. Am J Hum Biol. 2009;21(5):657-63. https://doi.org/10.1002/ajhb.20883. 
35. Doyle LW. Growth and respiratory health in adolescence of the extremely low-birth weight survivor. Clin Perinatol. 2000;27(2):421-32. https://doi.org/1 0.1016/S0095-5108(05)70029-4.

36. Ntenda PAM, Chuang Y-C. Analysis of individual-level and community-level effects on childhood undernutrition in Malawi. Pediatr Neonatol. 2018;59(4): 380-9. https://doi.org/10.1016/j.pedneo.2017.11.019.

37. Black RE, Laxminarayan R, Temmerman M, Walker N. Reproductive, maternal, newborn, and child health: disease control priorities, (volume 2); 2016. https://doi.org/10.1596/978-1-4648-0348-2.

38. Khan JR, Islam MM, Awan N, Muurlink O. Analysis of low birth weight and its co-variants in Bangladesh based on a sub-sample from nationally representative survey. BMC Pediatr. 2018;18(1):1-9. https://doi.org/10.1186/ s12887-018-1068-0.

39. Ikeda N, Irie Y, Shibuya K. Determinants of reduced child stunting in Cambodia: analysis of pooled data from three demographic and health surveys. Bull World Health Organ. 2013;91(5):341-9. https://doi.org/10.2471/BLT.12.113381.

40. Khanal V, Sauer K, Zhao Y. Determinants of complementary feeding practices among Nepalese children aged 6-23 months: findings from demographic and health survey 2011. BMC Pediatr. 2013;13(1):1-13. https:// doi.org/10.1186/1471-2431-13-131.

41. Conger RD, Conger KJ, Martin MJ. Socioeconomic status, family processes, and individual development. J Marriage Fam. 2010;72(3):685-704. https:// doi.org/10.1111/j.1741-3737.2010.00725.x.

42. Kochupurackal SU, Channa Basappa Y, Vazhamplackal SJ, Srinivas PN. An intersectional analysis of the composite index of anthropometric failures in India. Int J Equity Health. 2021;20(1):155. https://doi.org/10.1186/s1293 9-021-01499-y.

43. Das $\mathrm{S}$, Gulshan J. Different forms of malnutrition among under five children in Bangladesh: a cross sectional study on prevalence and determinants. BMC Nutrition. 2017;3(1):1. https://doi.org/10.1186/s40795-016-0122-2.

44. Khattak UK, lqbal SP, Ghazanfar H. The Role of Parents' Literacy in Malnutrition of Children Under the Age of Five Years in a Semi-Urban Community of Pakistan: A Case-Control Study. Cureus. 2017;9(6):e1316. https://doi.org/10.7759/cureus.1316.

45. Makoka D. The impact of maternal education on child nutrition: evidence from Malawi, Tanzania, and Zimbabwe. Calverton, Maryland: ICF International; 2013. http://dhsprogram.com/pubs/pdf/WP84/WP84.pdf

46. Murarkar S, Gothankar J, Doke P, Pore P, Lalwani S, Dhumale G, et al Prevalence and determinants of undernutrition among under-five children residing in urban slums and rural area, Maharashtra, India: a communitybased cross-sectional study. BMC Public Health. 2020;20(1):1-9. https://doi. org/10.1186/s12889-020-09642-0.

47. Kandala N-B, Madungu TP, Emina JB, Nzita KP, Cappuccio FP. Malnutrition among children under the age of five in the Democratic Republic of Congo (DRC): does geographic location matter? BMC Public Health. 2011;11:1-15.

48. Seid A, Seyoum B, Mesfin F. Determinants of acute malnutrition among children aged 6-59 months in public health facilities of pastoralist community, Afar region, Northeast Ethiopia: a case control study. J Nutr Metab. 2017;2017:1-7. https://doi.org/10.1155/2017/7265972.

49. Khanam M, Shimul SN, Sarker AR. Individual-, household-, and communitylevel determinants of childhood undernutrition in Bangladesh. Health Serv Res Manag Epidemiol. 2019;6:2333392819876555. https://doi.org/10.11 77/2333392819876555

50. Adhikari RP, Shrestha ML, Acharya A, Upadhaya N. Determinants of stunting among children aged 0-59 months in Nepal: findings from Nepal demographic and health survey, 2006, 2011, and 2016. BMC nutrition. 2019; 5(1):1-10. https://doi.org/10.1186/s40795-019-0300-0.

51. Kismul H, Acharya P, Mapatano MA, Hatløy A. Determinants of childhood stunting in the Democratic Republic of Congo: further analysis of demographic and health survey 2013-14. BMC Public Health. 2018;18(1):114. https://doi.org/10.1186/s12889-017-4621-0.

52. Sinha RK, Dua R, Bijalwan V, Rohatgi S, Kumar P. Determinants of stunting, wasting, and underweight in five high-burden pockets of four Indian states. Indian J Community Med. 2018;43:279.

53. Black RE, Morris SS, Bryce J. Where and why are 10 million children dying every year? Lancet. 2003;361(9376):2226-34. https://doi.org/10.1016/S01406736(03)13779-8

54. Nguyen PH, Avula R, Headey D, Tran LM, Ruel MT, Menon P. Progress and inequalities in infant and young child feeding practices in India between 2006 and 2016. Matern Child Nutr. 2018;14(S4):e12663. https://doi.org/1 $0.1111 / \mathrm{mcn} .12663$
55. Ahmed T, Mahfuz M, Ireen S, Ahmed AMS, Rahman S, Islam MM, et al. Nutrition of children and women in Bangladesh: trends and directions for the future. J Health Popul Nutr. 2012;30(1):1-11. https://doi.org/10.3329/ jhpn.v30i1.11268.

56. Smith LC, Ramakrishnan U, Ndiaye A, Haddad L, Martorell R. The importance of Women's status for child nutrition in developing countries: international food policy research institute (Ifpri) research report abstract 131. Food Nutr Bull. 2003;24(3):287-8. https://doi.org/10.1177/156482650302400309.

57. Biadgilign S, Shumetie A, Yesigat $H$. Does economic growth reduce childhood undernutrition in Ethiopia? PLoS One. 2016;11(8):e0160050. https://doi.org/10.1371/journal.pone.0160050.

58. UNICEF. Improving child nutrition: the achievable imperative for global progress. UN Children's Fund; 2013. https://reliefweb.int/report/world/ improving-child-nutrition-achievable-imperative-global-progress.

\section{Publisher's Note}

Springer Nature remains neutral with regard to jurisdictional claims in published maps and institutional affiliations.
Ready to submit your research? Choose BMC and benefit from:

- fast, convenient online submission

- thorough peer review by experienced researchers in your field

- rapid publication on acceptance

- support for research data, including large and complex data types

- gold Open Access which fosters wider collaboration and increased citations

- maximum visibility for your research: over $100 \mathrm{M}$ website views per year

At BMC, research is always in progress.

Learn more biomedcentral.com/submissions 